\title{
Note on the congruence of Ankeny-Artin-Chowla type modulo $p^{2}$
}

by

\author{
Stanislav Jakubec (Bratislava)
}

The results of [2] on the congruence of Ankeny-Artin-Chowla type modulo $p^{2}$ for real subfields of $\mathbb{Q}\left(\zeta_{p}\right)$ of a prime degree $l$ is simplified. This is done on the basis of a congruence for the Gauss period (Theorem 1). The results are applied for the quadratic field $\mathbb{Q}(\sqrt{p}), p \equiv 5(\bmod 8)$ (Corollary 1$)$.

\section{Notations}

- $B_{n}, E_{n}$ - Bernoulli and Euler numbers,

- $C_{n}=\frac{2^{n+1}\left(1-2^{n+1}\right) B_{n+1}}{n+1}$,

- $Q_{2}=\frac{2^{p-1}-1}{p}-$ Fermat quotient,

- $W_{p}=\frac{1+(p-1) !}{p}-$ Wilson quotient,

- $A_{n}=1+\frac{1}{2}+\ldots+\frac{1}{n}, \quad A_{0}=0$.

Introduction. In [2] the congruence of Ankeny-Artin-Chowla type modulo $p^{2}$ for real subfields of the field $\mathbb{Q}\left(\zeta_{p}\right)$ of prime degree $l$ is proved. The following notation and theorem are taken from [2].

Let $a$ be a fixed primitive root modulo $p$, let $\chi$ be the Dirichlet character of order $n, n \mid p-1, \chi(x)=\zeta_{n}^{\operatorname{ind}_{a} x}$. Let $g$ be such that $g \equiv a^{(p-1) / n}(\bmod p)$ and $g^{n} \equiv 1\left(\bmod p^{p}\right)$. Denote by $\mathfrak{p}$ a prime divisor of $\mathbb{Q}\left(\zeta_{n}\right)$ such that $\mathfrak{p} \mid p$ and $1 / g \equiv \zeta_{n}\left(\bmod \mathfrak{p}^{p}\right)$.

Define the rational numbers $A_{0}(n), A_{1}(n), \ldots, A_{n-1}(n)$ by

$$
A_{0}(n)=-1 / n,
$$

$\tau\left(\chi^{i}\right)^{n} \equiv n^{n} A_{i}(n)^{n}(-p)^{i}\left(\bmod \mathfrak{p}^{2+i}\right), \quad A_{i}(n) \equiv \frac{(p-1) / n}{(i(p-1) / n) !}(\bmod p)$, where $\tau(\chi)$ is the Gauss sum.

1991 Mathematics Subject Classification: Primary 11R29. 
Put $m=(p-1) / 2$, and

$$
\begin{gathered}
G_{j}(X)=A_{0}(m) X^{j}+A_{1}(m) X^{j-1}+\ldots+A_{j}(m), \\
F_{j}(X)=\frac{1}{(p-1) !} X^{j}+\frac{1}{(p+1) !} X^{j-1}+\frac{1}{(p+3) !} X^{j-2}+\ldots+\frac{1}{(p+2 j-1) !} .
\end{gathered}
$$

Define

$$
E_{n}^{*}=\frac{E_{2 n}}{(2 n) !} \quad \text { for } n=1,2,3, \ldots,
$$

where $E_{2 n}$ are the Euler numbers, i.e. $E_{0}=1, E_{2}=-1, E_{4}=5, E_{6}=$ $-61, E_{8}=1385, E_{10}=-50521, E_{12}=2702765, E_{14}=-199360981, \ldots$

Consider the formal expressions $G_{j}\left(E^{*}\right)$ and $F_{j}\left(E^{*}\right)$, where

$$
\left(E^{*}\right)^{k}=E_{k}^{*} \text {. }
$$

Let $\beta_{0}, \beta_{1}, \ldots, \beta_{l-1}$ be the integral basis of the field $K$ formed by the Gauss periods. Let $\delta$ be the unit

$$
\delta=x_{0} \beta_{0}+x_{1} \beta_{1}+\ldots+x_{l-1} \beta_{l-1} .
$$

Associate with the unit $\delta$ the polynomial $f(X)$ as follows:

$$
f(X)=X^{l-1}+d_{1} X^{l-2}+d_{2} X^{l-3}+\ldots+d_{l-1},
$$

where

$$
d_{i}=-l A_{i}(l) \frac{x_{0}+x_{1} g^{i}+x_{2} g^{2 i}+\ldots+x_{l-1} g^{i(l-1)}}{x_{0}+x_{1}+\ldots+x_{l-1}}
$$

for $i=1, \ldots, l-1$. Put $S_{j}=S_{j}\left(d_{1}, \ldots, d_{l-1}\right)=$ sum of $j$ th powers of the roots of $f(X)$ for $j=1, \ldots, 2 l-1$. Hence

$$
S_{1}=-d_{1}, \quad S_{2}=d_{1}^{2}-2 d_{2}, \quad S_{3}=-d_{1}^{3}+3 d_{1} d_{2}-3 d_{3}, \ldots
$$

Define the numbers $T_{1}, \ldots, T_{2 l-1}$ as follows:

$$
\begin{aligned}
T_{i}= & -\frac{1}{(i(p-1) / l) !} 2^{i(p-1) / l-1}\left(2^{i(p-1) / l}-1\right) B_{i(p-1) / l} \\
& -i \frac{p-1}{4 l} G_{i(p-1) /(2 l)}\left(E^{*}\right)
\end{aligned}
$$

for $i=1, \ldots, l-1$, and

$$
\begin{aligned}
T_{l}= & \frac{1-Q_{2}}{2}, \quad \text { where } Q_{2}=\frac{2^{p-1}-1}{p} \\
T_{l+i}= & -\frac{1}{\left(p-1+i \frac{p-1}{l}\right) !} \\
& \times 2^{p-1+i(p-1) / l-1}\left(2^{p-1+i(p-1) / l}-1\right) B_{(p-1+i(p-1) / l)} \\
& +\left(\frac{p-1}{2}+i \frac{p-1}{2 l}\right) F_{i(p-1) /(2 l)}\left(E^{*}\right)
\end{aligned}
$$

for $i=1, \ldots, l-1$. 
Define

$$
\alpha_{i}=c_{0}+c_{1} g^{i}+c_{2} g^{2 i}+\ldots+c_{l-2} g^{(l-2) i}
$$

for $i=1, \ldots, 2 l-1$.

Let $X_{1}, \ldots, X_{2 l-1} \in \mathbb{Q}$ and let

$$
g(X)=X^{2 l-1}+Y_{1} X^{2 l-2}+\ldots+Y_{2 l-1}
$$

be a polynomial such that

$$
X_{j}=\text { sum of the } j \text { th powers of the roots of } g(X) .
$$

Define the mapping $\Phi: \mathbb{Q}^{2 l-1} \rightarrow \mathbb{Q}^{l}$ as follows:

$$
\Phi\left(X_{1}, \ldots, X_{2 l-1}\right)=\left(1-p Y_{l}, Y_{1}-p Y_{l+1}, \ldots, Y_{l-1}-p Y_{2 l-1}\right) .
$$

TheOREM 1 of [2]. Let $l$ and $p$ be primes with $p \equiv 1(\bmod l)$ and let $K \subset \mathbb{Q}\left(\zeta_{p}+\zeta_{p}^{-1}\right)$ with $[K: \mathbb{Q}]=l$. Suppose that 2 is not an lth power modulo $p$. Let $\delta$ be a unit of $K$ such that $\left[U_{K}:\langle\delta\rangle\right]=f,(f, p)=1$. Let $\eta_{2}^{f}=\delta^{c_{0}} \sigma(\delta)^{c_{1}} \ldots \sigma^{l-2}(\delta)^{c_{l-2}}$ and $\alpha_{i}=c_{0}+c_{1} g^{i}+c_{2} g^{2 i}+\ldots+c_{l-2} g^{(l-2) i}$ for $i=1, \ldots, 2 l-1$. The following congruence holds:

(3) $\varepsilon\left(\frac{x_{0}+x_{1}+\ldots+x_{l-1}}{-l}\right)^{\alpha_{l}} \Phi\left(\alpha_{1} S_{1}, \ldots, \alpha_{2 l-1} S_{2 l-1}\right)$

$$
\equiv(2+2 p)^{f(p-1) /(2 l)} \Phi\left(f T_{1}, \ldots, f T_{2 l-1}\right)\left(\bmod p^{2}\right),
$$

where $\varepsilon= \pm 1$.

This theorem is applied to the real quadratic field.

The quadratic case: $K=\mathbb{Q}(\sqrt{p}), p \equiv 5(\bmod 8)$ and $T+U \sqrt{p}>1$ is the fundamental unit. By [2] we have

$$
S_{1}=2 A_{1}(2) \frac{U}{T}, \quad S_{2}=-\frac{U^{2}}{T^{2}}, \quad S_{3}=-2 A_{1}(2) \frac{U^{3}}{T^{3}} .
$$

For the numbers $T_{1}, T_{2}, T_{3}$ we have

$$
\begin{aligned}
T_{1}= & -\frac{1}{((p-1) / 2) !} 2^{(p-1) / 2-1}\left(2^{(p-1) / 2}-1\right) B_{(p-1) / 2}-\frac{p-1}{8} G_{(p-1) / 4}\left(E^{*}\right), \\
T_{2}= & \frac{1}{2}\left(1-Q_{2}\right), \\
T_{3}= & -\frac{1}{(3(p-1) / 2) !} 2^{3(p-1) / 2-1}\left(2^{3(p-1) / 2}-1\right) B_{3(p-1) / 2} \\
& +\frac{3(p-1)}{4} F_{(p-1) / 4}\left(E^{*}\right) .
\end{aligned}
$$

It is easy to see that $\Phi\left(X_{1}, X_{2}, X_{3}\right)=\left(1-p \frac{X_{1}^{2}-X_{2}}{2},-X_{1}-p\left(-\frac{1}{6} X_{1}^{3}+\frac{1}{2} X_{1} X_{2}-\frac{1}{3} X_{3}\right)\right)$. 
Hence

$$
\varepsilon T^{h} \Phi\left(h S_{1}, h S_{2}, h S_{3}\right) \equiv(2+2 p)^{(p-1) / 4} \Phi\left(T_{1}, T_{2}, T_{3}\right)\left(\bmod p^{2}\right) .
$$

The greatest difficulty in applying Theorem 1 of [2] to fields of concrete degrees $l=2,3, \ldots$ is caused by the fact that the numbers $A_{i}(n), G_{j}\left(E^{*}\right)$ and $F_{j}\left(E^{*}\right)$ are defined in a very complicated way. This constraint appears also in the case of a quadratic field, because of the unclear values $G_{(p-1) / 4}\left(E^{*}\right)$ and $F_{(p-1) / 4}\left(E^{*}\right)$ involved.

The aim of this paper is to eliminate the above mentioned constraints. This will be done on the basis of a congruence for the Gauss period (Theorem 1 ). The results will be applied to the real quadratic field $\mathbb{Q}(\sqrt{p}), p \equiv 5$ $(\bmod 8)$. In this case we get a simple congruence modulo $p^{2}$ (Corollary 1 ) involving: the fundamental unit $T+U \sqrt{p}$, the class number $h$, the Bernoulli numbers $B_{(p-1) / 2}, B_{3(p-1) / 2}$ and the Fermat quotient $Q_{2}$.

1. Congruence for the Gauss period. Let $p \equiv 1(\bmod n)$ be prime and let $K$ be a subfield of the field $\mathbb{Q}\left(\zeta_{p}\right)$ of the degree $n$ over $\mathbb{Q}$. Let $a$ be a primitive root modulo $p$. We consider the automorphism $\sigma$ of the field $\mathbb{Q}\left(\zeta_{p}\right)$ such that $\sigma\left(\zeta_{p}\right)=\zeta_{p}^{a}$.

Further we denote:

$$
\begin{gathered}
\beta_{0}=\operatorname{Tr}_{\mathbb{Q}\left(\zeta_{p}\right) / K}\left(\zeta_{p}\right) ; \quad \beta_{i}=\sigma^{i}\left(\beta_{0}\right) \quad \text { for } i=1, \ldots, n-1 ; \\
k=(p-1) / n ; \quad a^{k} \equiv g(\bmod p) .
\end{gathered}
$$

In [3] the following theorem is proved:

TheOREM 1 of [3]. There is a number $\pi \in K$ with $\pi \mid p$ such that

(i) $N_{K / \mathbb{Q}}(\pi)=(-1)^{n} p$,

(ii) $\sigma(\pi) \equiv g \pi\left(\bmod \pi^{n+1}\right)$,

(iii) $\beta_{0} \equiv k \sum_{i=0}^{n} \frac{1}{(k i) !} \pi^{i}\left(\bmod \pi^{n+1}\right)$.

In [4], it is proved that for any $t$ there exists $\pi \in K$ such that

$$
\sigma \pi \equiv g \pi\left(\bmod \pi^{t n+1}\right), \quad \text { where } g^{n} \equiv 1\left(\bmod p^{t}\right) .
$$

Hence

$$
\beta_{0} \equiv \sum_{i=0}^{t n} a_{i} \pi^{i}\left(\bmod \pi^{t n+1}\right), \quad 0 \leq a_{i}<p .
$$

Because $\pi^{n} \equiv-p\left(\bmod \pi^{t n+1}\right)$, the congruence (1) can be rewritten as

$$
\beta_{0} \equiv \sum_{i=0}^{n-1} a_{i}^{*} \pi^{i}\left(\bmod \pi^{t n+1}\right),
$$

where $a_{i}^{*}=a_{i}-p a_{i+n}+p^{2} a_{i+2 n}+\ldots$ 
Hence for any divisor $n$ of $p-1, n \neq 1$, there are numbers $a_{0}^{*}, a_{1}^{*}, \ldots, a_{n-1}^{*}$ such that

(i) $a_{i}^{*} \equiv \frac{k}{(k i) !}(\bmod p)$,

(ii) $\beta_{0} \equiv \sum_{i=0}^{n-1} a_{i}^{*} \pi^{i}\left(\bmod \pi^{S n+1}\right)$ for any exponent $S$.

Lemma 1. Let $p$ be a prime and let $n$ be a divisor of $p-1, n \neq 1$. There exists a prime divisor $\mathfrak{p}$ of the field $\mathbb{Q}\left(\zeta_{n}\right)$ with $\mathfrak{p} \mid p$ such that for any exponent $S$ the following holds:

(i) $a_{i}^{*} \equiv \frac{k}{(k i) !}(\bmod p)$ for $i=1, \ldots, n-1$,

(ii) $\tau\left(\chi^{i}\right) \equiv n a_{i}^{*} \pi^{i}\left(\bmod \mathfrak{p}^{S}\right)$.

Proof. Take $S$ and $\pi$ such that

$$
\sigma \pi \equiv g \pi\left(\bmod \pi^{(S+1) n+1}\right), \quad g^{n} \equiv 1\left(\bmod p^{S+1}\right) .
$$

Then

$$
\beta_{0} \equiv \sum_{i=0}^{n-1} a_{i}^{*} \pi^{i}\left(\bmod \pi^{(S+1) n+1}\right)
$$

hence

$$
\begin{aligned}
& \frac{1}{\pi^{i}}\left(\beta_{0}-\left(a_{0}^{*}+a_{1}^{*} \pi+\ldots+a_{i-1}^{*} \pi^{i-1}\right)\right) \\
& \quad \equiv a_{i}^{*}+a_{i+1}^{*} \pi+\ldots+a_{n-1}^{*} \pi^{n-1-i}\left(\bmod \pi^{(S+1) n+1-i}\right) .
\end{aligned}
$$

Now take the trace $\operatorname{Tr}_{K / \mathbb{Q}}$ of the right and left sides. For $0<i<n$ we have

$$
\operatorname{Tr}_{K / \mathbb{Q}}\left(A \pi^{i}\right) \equiv 0\left(\bmod \pi^{(S+1) n+1}\right)
$$

It follows that

$$
\begin{aligned}
& \frac{1}{\pi^{i}}\left(\beta_{0}+\frac{1}{g^{i}} \sigma \beta_{0}+\frac{1}{g^{2 i}} \sigma^{2} \beta_{0}+\ldots+\frac{1}{g^{(n-1) i}} \sigma^{n-1} \beta_{0}\right) \\
& \equiv n a_{i}^{*}\left(\bmod \pi^{(S+1) n+1-i}\right) .
\end{aligned}
$$

Because $g^{n} \equiv 1\left(\bmod p^{S+1}\right)$, there exists a prime divisor $\mathfrak{p}$ of the field $\mathbb{Q}\left(\zeta_{n}\right)$ with $\mathfrak{p} \mid p$ such that

$$
1 / g \equiv \zeta_{n}\left(\bmod \mathfrak{p}^{S+1}\right)
$$

Hence

$$
\beta_{0}+\frac{1}{g^{i}} \sigma \beta_{0}+\frac{1}{g^{2 i}} \sigma^{2} \beta_{0}+\ldots+\frac{1}{g^{(n-1) i}} \sigma^{n-1} \beta_{0} \equiv \tau\left(\chi^{i}\right)\left(\bmod \mathfrak{p}^{S+1}\right) .
$$

Because $\pi^{n} \approx p$, we have $\tau\left(\chi^{i}\right) \equiv n a_{i}^{*} \pi^{i}\left(\bmod \mathfrak{p}^{S}\right)$. 
The following theorem gives a congruence for the Gauss period modulo $\pi^{2 n+1}$. For simplicity, the coefficients are denoted by $a_{i}$ (instead of $a_{i}^{*}$ ).

THEOREM 1. Let $p$ be an odd prime and let $\pi$ be the above defined element of the field $\mathbb{Q}\left(\zeta_{p}\right)$. Then

$$
\zeta_{p} \equiv \frac{-1}{p-1}+a_{1} \pi+a_{2} \pi^{2}+\ldots+a_{p-2} \pi^{p-2}\left(\bmod \pi^{2(p-1)+1}\right),
$$

where

(i) $a_{i} \equiv \frac{1}{i !}+p \frac{1}{(i-1) !}\left(W_{p}-A_{i-1}\right)\left(\bmod p^{2}\right) \quad$ for $i=1, \ldots,(p-3) / 2$,

$$
a_{i} a_{p-1-i} \equiv(-1)^{i+1}(1+2 p)\left(\bmod p^{2}\right) .
$$

REMARK. On the basis of this theorem a congruence modulo $\pi^{2(p-1)+1}$ for any Gauss period $\beta, \beta \in K$, can be given. This follows from the fact that $\beta=\operatorname{Tr}_{\mathbb{Q}\left(\zeta_{p}\right) / K}\left(\zeta_{p}\right)$.

Pro of (of Theorem 1). Clearly

$$
\operatorname{Tr}_{\mathbb{Q}\left(\zeta_{p}\right) / \mathbb{Q}}\left(\zeta_{p}\right)=-1 \equiv(p-1) a_{0}\left(\bmod \pi^{2(p-1)+1}\right),
$$

hence $a_{0} \equiv \frac{-1}{p-1}\left(\bmod \pi^{2(p-1)+1}\right)$.

The congruence (ii) is proved as follows. By Lemma 1,

$$
\begin{gathered}
\tau\left(\chi^{i}\right) \equiv(p-1) a_{i} \pi^{i}\left(\bmod \pi^{2(p-1)+1}\right), \\
\tau\left(\chi^{p-1-i}\right) \equiv(p-1) a_{p-1-i} \pi^{p-1-i}\left(\bmod \pi^{2(p-1)+1}\right) .
\end{gathered}
$$

Hence

$$
\tau\left(\chi^{i}\right) \tau\left(\chi^{p-1-i}\right)=(-1)^{i} p \equiv(p-1)^{2} a_{i} a_{p-1-i}(-p)\left(\bmod \pi^{2(p-1)+1}\right),
$$

and we have (ii).

Now we prove (i). Since $\zeta_{p}^{2}=\sigma_{2}\left(\zeta_{p}\right)$ we have

$$
\begin{aligned}
& \left(1+p+p^{2}+a_{1} \pi+a_{2} \pi^{2}+\ldots+a_{p-2} \pi^{p-2}\right)^{2} \\
& \equiv 1+p+p^{2}+a_{1} 2^{p} \pi+a_{2} 2^{2 p} \pi^{2}+\ldots+a_{p-2} 2^{p(p-2)} \pi^{p-2}\left(\bmod \pi^{2(p-1)+1}\right) .
\end{aligned}
$$

Let us write the numbers $a_{i}$ in the form

$$
a_{i}=\frac{1}{i !}+x_{i} p \quad \text { for } i=1, \ldots,(p-3) / 2 .
$$

Squaring the left-hand side we get

$$
\left(1+p+p^{2}\right)^{2}+c_{1} \pi+c_{2} \pi^{2}+\ldots+c_{p-2} \pi^{p-2},
$$

where

$$
\begin{aligned}
& c_{1}=2\left(1+p+p^{2}\right)\left(1+x_{1} p\right), \\
& c_{2}=2\left(1+p+p^{2}\right)\left(\frac{1}{2 !}+x_{2} p\right)+\left(1+x_{1} p\right)^{2},
\end{aligned}
$$




$$
c_{3}=2\left(1+p+p^{2}\right)\left(\frac{1}{3 !}+x_{3} p\right)+2\left(1+x_{1} p\right)\left(\frac{1}{2 !}+x_{2} p\right), \ldots
$$

The coefficient of $\pi^{p-1}$ (after squaring the left-hand side) is

$$
\sum_{i=1}^{p-2} a_{i} a_{p-1-i} \equiv 1+2 p\left(\bmod p^{2}\right)
$$

which follows from the congruence (ii).

It is easy to see that it is sufficient to consider the coefficients of $\pi^{p}$, $\pi^{p+1}, \pi^{p+2}, \ldots$ modulo $p$.

The coefficient of $\pi^{p}$ is

$$
\sum_{i=2}^{p-2} a_{i} a_{p-i} \equiv \sum_{i=2}^{p-2} \frac{1}{i !} \cdot \frac{1}{(p-i) !} \equiv \frac{1}{p !} \sum_{i=2}^{p-2}\left(\begin{array}{c}
p \\
i
\end{array}\right) \equiv-\frac{1}{p}\left(2^{p}-2-2 p\right)(\bmod p) .
$$

Let $d_{p+k}$ be the coefficient of $\pi^{p+k}$ for $k>0$. Then

$$
d_{p+k} \equiv \frac{-1}{p} \cdot \frac{1}{k !}\left(2^{p+k}-2 \sum_{i=0}^{k+1}\left(\begin{array}{c}
p+k \\
i
\end{array}\right)\right)(\bmod p) .
$$

Since $\pi^{p-1} \equiv-p\left(\bmod \pi^{2(p-1)+1}\right)$, we have

$$
\begin{aligned}
1+2 p+3 p^{2} & +c_{1} \pi+c_{2} \pi^{2}+\ldots+c_{p-2} \pi^{p-2} \\
& -p\left(1+2 p-2\left(Q_{2}-1\right) \pi+d_{p+1} \pi^{2}+\ldots+d_{p+p-3} \pi^{p-2}\right) \\
\equiv & 1+p+p^{2}+2^{p}\left(1+x_{1} p\right) \pi+2^{2 p}\left(\frac{1}{2 !}+x_{2} p\right) \pi^{2} \\
& +\ldots+2^{(p-2) p}\left(\frac{1}{(p-2) !}+x_{p-2}\right) \pi^{p-2}\left(\bmod \pi^{2(p-1)+1}\right) .
\end{aligned}
$$

It follows that

$$
\begin{gathered}
\left(c_{2}-p d_{p+1}-2^{2 p}\left(\frac{1}{2 !}+x_{2} p\right)\right) \pi^{2}+\left(c_{3}-p d_{p+2}-2^{3 p}\left(\frac{1}{3 !}+x_{3} p\right)\right) \pi^{3}+\ldots \\
\equiv 0\left(\bmod \pi^{2(p-1)+1}\right) .
\end{gathered}
$$

Hence the coefficients of $\pi^{2}, \pi^{3}, \ldots$ must be divisible by $p$. After reducing by $p$ we get

$$
\begin{array}{r}
\frac{c_{2}-p d_{p+1}-2^{2 p}\left(\frac{1}{2 !}+x_{2} p\right)}{p} \pi^{2}+\frac{c_{3}-p d_{p+2}-2^{3 p}\left(\frac{1}{3 !}+x_{3}\right)}{p} \pi^{3}+\ldots \\
\quad \equiv 0\left(\bmod \pi^{p-1+1}\right),
\end{array}
$$

hence

$$
\frac{c_{2}-p d_{p+1}-2^{2 p}\left(\frac{1}{2 !}+x_{2} p\right)}{p} \equiv 0(\bmod p)
$$




$$
\frac{c_{3}-p d_{p+2}-2^{3 p}\left(\frac{1}{3 !}+x_{3} p\right)}{p} \equiv 0(\bmod p),
$$

etc.

Substituting for $c_{2}$ and reducing we have

$$
\frac{2^{2}}{2 !} \cdot \frac{1-2^{2(p-1)}}{p}+\frac{2}{2 !}+2 x_{1}+\left(2-2^{2}\right) x_{2}-d_{p+1} \equiv 0(\bmod p) .
$$

Continuing, we find that $x_{1}, x_{2}, \ldots, x_{(p-3) / 2}$ satisfy the system of linear equations modulo $p$ with matrix

$$
\left(\begin{array}{cccccccc}
1 & 1-2 & 0 & & \cdots & & 0 \\
\frac{1}{2 !} & \frac{1}{1 !} & 1-2^{2} & 0 & 0 & \ldots & 0 \\
\frac{1}{3 !} & \frac{1}{2 !} & \frac{1}{1 !} & 1-2^{3} & 0 & \ldots & 0 \\
\vdots & & & & & & \\
\frac{1}{((p-3) / 2) !} & \frac{1}{((p-5) / 2) !} & \ldots & \frac{1}{3 !} & \frac{1}{2 !} & 1 & 1-2^{(p-3) / 2}
\end{array}\right),
$$

and right-hand side consisting of the numbers $r_{k}$ satisfying

$$
2 r_{k}=d_{p+k}+\frac{1}{(k+1) !} 2^{k+1} \frac{2^{(k+1)(p-1)}-1}{p}-\frac{2}{(k+1) !},
$$

where

$$
d_{p+k} \equiv \frac{-1}{p} \cdot \frac{1}{k !}\left(2^{p+k}-2 \sum_{i=0}^{k+1}\left(\begin{array}{c}
p+k \\
i
\end{array}\right)\right)(\bmod p) .
$$

For $1 \leq i$ the following congruence holds:

$$
\left(\begin{array}{c}
p+k \\
i
\end{array}\right) \equiv\left(\begin{array}{c}
k \\
i
\end{array}\right)\left(1+p\left(\frac{1}{k}+\frac{1}{k+1}+\ldots+\frac{1}{k-i+1}\right)\right)\left(\bmod p^{2}\right) .
$$

From this we get

$$
\begin{aligned}
& \sum_{i=0}^{k+1}\left(\begin{array}{c}
p+k \\
i
\end{array}\right) \equiv 2^{k}+\frac{1}{k+1} \\
& +p\left(A_{k}+\left(\begin{array}{c}
k \\
k-1
\end{array}\right)\left(A_{k}-A_{1}\right)+\left(\begin{array}{c}
k \\
k-2
\end{array}\right)\left(A_{k}-A_{2}\right)+\ldots\right. \\
& \left.+\left(\begin{array}{l}
k \\
1
\end{array}\right)\left(A_{k}-A_{k-1}\right)\right)\left(\bmod p^{2}\right) \text {. }
\end{aligned}
$$

After rearrangements we have

$$
r_{k} \equiv \frac{1}{k !}\left(2^{k} A_{k}-\sum_{i=1}^{k}\left(\begin{array}{c}
k \\
i
\end{array}\right) A_{i}\right)(\bmod p) .
$$


Put

$$
x_{i}=\frac{x_{1}}{(i-1) !}-\frac{A_{i-1}}{(i-1) !} \quad \text { for } i=1, \ldots,(p-3) / 2 .
$$

For each $n=1, \ldots,(p-3) / 2$ we obtain

$$
\begin{aligned}
\frac{1}{n !} x_{1}+ & \frac{1}{(n-1) !}\left(\frac{x_{1}}{1 !}-\frac{A_{1}}{1 !}\right)+\frac{1}{(n-2) !}\left(\frac{x_{1}}{2 !}-\frac{A_{2}}{2 !}\right) \\
& +\ldots+\frac{1}{1 !}\left(\frac{x_{1}}{(n-1) !}-\frac{A_{n-1}}{(n-1) !}\right)+\left(1-2^{n}\right)\left(\frac{x_{1}}{n !}-\frac{A_{n}}{n !}\right) \\
= & \frac{1}{n !} x_{1}\left(\left(\begin{array}{c}
n \\
0
\end{array}\right)+\left(\begin{array}{c}
n \\
1
\end{array}\right)+\ldots+\left(\begin{array}{c}
n \\
n
\end{array}\right)\right)-\frac{2^{n}}{n !} x_{1}-\frac{1}{n !} \sum_{i=1}^{n}\left(\begin{array}{c}
n \\
i
\end{array}\right) A_{i}+\frac{2^{n}}{n !} A_{n} \\
= & r_{n} .
\end{aligned}
$$

Hence the numbers $x_{1}, x_{2}, \ldots, x_{(p-3) / 2}$, where

$$
x_{i}=\frac{x_{1}}{(i-1) !}-\frac{A_{i-1}}{(i-1) !} \quad \text { for } i=1, \ldots,(p-3) / 2,
$$

are the solution of the system of equations considered. It remains to determine $x_{1}$. Consider the coefficient $a_{2}$,

$$
a_{2}=\frac{1}{2 !}+x_{2} p=\frac{1}{2 !}+p\left(x_{1}-1\right) .
$$

By Theorem 5 of [4],

$$
\begin{aligned}
& \zeta_{p}+\zeta_{p}^{-1} \\
& \equiv 2\left(1+p+p^{2}\right)+\left(\frac{2}{2 !}-2 p \frac{p-1-p(p+1) B_{p-1}}{p}\right) \pi_{1}+\ldots\left(\bmod \pi_{1}^{2 m+1}\right),
\end{aligned}
$$

where $m=(p-1) / 2$ and $\pi_{1}=\pi^{2}$. It follows that

$$
2 a_{2}=1+2 p\left(x_{1}-1\right) \equiv 1-2 p \frac{p-1-p(p+1) B_{p-1}}{p}\left(\bmod p^{2}\right),
$$

hence

$$
x_{1} \equiv \frac{1+p(p+1) B_{p-1}}{p} \equiv W_{p}(\bmod p)
$$

2. Applications. Define $N=(p-1)+i \frac{p-1}{l}, n=i \frac{p-1}{l}-1$.

TheOREM 2. For the number $T_{i}$ the following congruences hold:

(i) $T_{l+i} \equiv \frac{N}{2 n !}\left(-\frac{C_{N-1}-C_{n}}{p}+A_{n} C_{n}+\sum_{i=0}^{n-1}\left(\begin{array}{c}n \\ i\end{array}\right) \frac{C_{i}}{n-i}-\frac{E_{n+1}}{n+1}\right)$

$(\bmod p)$, 
(ii) $\quad T_{i} \equiv \frac{C_{n}}{2 n !}-i \frac{p(p-1)}{2 \ln !}\left(\frac{E_{n+1}}{n+1}-W_{p} C_{n}-\sum_{i=0}^{n-1}\left(\begin{array}{c}n \\ i\end{array}\right) \frac{C_{i}}{n-i}\right)\left(\bmod p^{2}\right)$ for $i=1, \ldots, l-1$.

Proof. To determine $T_{l+i}$ it is necessary to determine the sum

$$
F_{j}\left(E^{*}\right)=\frac{1}{(p-1) !} \cdot \frac{E_{2 j}}{(2 j) !}+\frac{1}{(p+1) !} \cdot \frac{E_{2 j-2}}{(2 j-2) !}+\ldots+\frac{1}{(p+2 j-1) !},
$$

where $j=(n+1) / 2$.

We have

$$
\begin{aligned}
p F_{j}\left(E^{*}\right)= & \frac{p}{(p-1) !} \cdot \frac{E_{2 j}}{(2 j) !} \\
& +\frac{1}{(p-1) !}\left(\frac{E_{2 j-2}}{(p+1)(2 j-2) !}+\frac{E_{2 j-4}}{(p+1)(p+2)(p+3)(2 j-4) !}\right. \\
& \left.+\ldots+\frac{1}{(p+1)(p+2) \ldots(p+2 j-1)}\right)\left(\bmod p^{2}\right) .
\end{aligned}
$$

Expressing the product $(p+1)(p+2) \ldots(p+i)$ modulo $p^{2}$ we get

$$
\begin{aligned}
p F_{j}\left(E^{*}\right)= & \frac{p}{(p-1) !} \cdot \frac{E_{2 j}}{(2 j) !} \\
& +\frac{1}{(p-1) !}\left(\frac{E_{2 j-2}}{(p+1)(2 j-2) !}+\frac{E_{2 j-4}}{3 !\left(1+p A_{3}\right)(2 j-4) !}\right. \\
& \left.+\ldots+\frac{1}{(2 j-1) !\left(1+p A_{2 j-1}\right)}\right)\left(\bmod p^{2}\right) .
\end{aligned}
$$

From $1 /(1+p k) \equiv 1-p k\left(\bmod p^{2}\right)$ we get

$$
\begin{aligned}
p F_{j}\left(E^{*}\right) \equiv & \frac{p}{(p-1) !} \cdot \frac{E_{2 j}}{(2 j) !} \\
& +\frac{1}{(p-1) !(2 j-1) !} \\
& \times\left(\left(\begin{array}{c}
2 j-1 \\
1
\end{array}\right) E_{2 j-2}+\left(\begin{array}{c}
2 j-1 \\
3
\end{array}\right) E_{2 j-4}+\ldots+1\right) \\
& -\frac{p}{(p-1) !(2 j-1) !}\left(\left(\begin{array}{c}
2 j-1 \\
1
\end{array}\right) E_{2 j-2} A_{1}+\left(\begin{array}{c}
2 j-1 \\
3
\end{array}\right) E_{2 j-4} A_{3}\right. \\
& \left.+\ldots+A_{2 j-1}\right)\left(\bmod p^{2}\right) .
\end{aligned}
$$

According to formula (51.1.2) of [1],

$$
\sum_{k=1}^{n}( \pm 1)^{k} \frac{(-n)_{k}}{k !} E_{k}=\frac{1}{n+1}(-2)^{n+1}\left(2^{n+1}-1\right) B_{n+1},
$$


we have

$\left(\begin{array}{c}2 j-1 \\ 1\end{array}\right) E_{2 j-2}+\left(\begin{array}{c}2 j-1 \\ 3\end{array}\right) E_{2 j-4}+\ldots+1=\frac{1}{2 j} 2^{2 j}\left(2^{2 j}-1\right) B_{2 j}=-C_{2 j-1}$.

Now summing up we get

Since

$$
\left(\begin{array}{c}
2 j-1 \\
1
\end{array}\right) E_{2 j-2} A_{1}+\left(\begin{array}{c}
2 j-1 \\
3
\end{array}\right) E_{2 j-4} A_{3}+\ldots+A_{2 j-1}
$$

$$
\begin{gathered}
\sum_{k=1}^{\infty} \frac{( \pm 1)^{k} A_{k}}{k !} x^{k}=e^{ \pm x}(C+\ln x-\operatorname{Ei}(\mp x)), \\
\sum_{k=1}^{\infty} \frac{( \pm 1)^{k}}{k k !} x^{k}=-C-\ln x+\operatorname{Ei}( \pm x),
\end{gathered}
$$

it follows that

Moreover,

$$
\sum_{k=1}^{\infty} \frac{(-1)^{k} A_{k}}{k !} x^{k}=-e^{-x} \sum_{k=1}^{\infty} \frac{1}{k k !} x^{k}
$$

$$
\frac{2}{e^{x}+e^{-x}}=1+\frac{E_{2}}{2 !} x^{2}+\frac{E_{4}}{4 !} x^{4}+\ldots,
$$

hence the generating function for the sum we looked for is

Hence

$$
\frac{2}{e^{2 x}+1} \sum_{k=1}^{\infty} \frac{1}{k k !} x^{k}, \quad \text { where } \quad \frac{2}{e^{x}+1}=\sum_{k=0}^{\infty} \frac{C_{k}}{k !} \cdot \frac{x^{k}}{2^{k}} .
$$

and it follows that

$$
\sum_{k=0}^{\infty} \frac{C_{k}}{k !} x^{k} \sum_{k=1}^{\infty} \frac{1}{k k !} x^{k}
$$

$$
\left(\begin{array}{c}
2 j-1 \\
1
\end{array}\right) E_{2 j-2} A_{1}+\left(\begin{array}{c}
2 j-1 \\
3
\end{array}\right) E_{2 j-4} A_{3}+\ldots+A_{2 j-1}=\sum_{i=0}^{n-1}\left(\begin{array}{c}
n \\
i
\end{array}\right) \frac{C_{i}}{n-i},
$$

where $n=2 j-1$. Therefore

$$
p F_{j}\left(E^{*}\right) \equiv \frac{-C_{n}}{n !(p-1) !}+\frac{p}{n !}\left(\sum_{i=0}^{n-1}\left(\begin{array}{c}
n \\
i
\end{array}\right) \frac{C_{i}}{n-i}-\frac{E_{n+1}}{n+1}\right)\left(\bmod p^{2}\right) .
$$

Hence

$$
\begin{aligned}
p T_{l+i}= & -\frac{p}{\left(p-1+i \frac{p-1}{l}\right) !} 2^{p-1+i(p-1) / l-1}\left(2^{p-1+i(p-1) / l}-1\right) B_{(p-1+i(p-1) / l)} \\
& +\left(\frac{p-1}{2}+i \frac{p-1}{2 l}\right)\left(\frac{-C_{n}}{n !(p-1) !}+\frac{p}{n !}\left(\sum_{i=0}^{n-1}\left(\begin{array}{c}
n \\
i
\end{array}\right) \frac{C_{i}}{n-i}-\frac{E_{n+1}}{n+1}\right)\right)
\end{aligned}
$$

for $i=1, \ldots, l-1$. 
Rearranging this congruence we get the congruence (i). The congruence (ii) is obtained using Theorem 1 by substituting $2+2 p, 2 a_{2}, 2 a_{4}, \ldots$ for $A_{0}(m), A_{1}(m), \ldots$, in the formula for $G_{j}\left(E^{*}\right), j=i(p-1) /(2 l)$.

Corollary 1. Let $p$ be a prime, $p \equiv 5(\bmod 8)$. Let $T+U \sqrt{p}>1$ be a fundamental unit and $h$ be the class number. Then:

$$
\begin{aligned}
& \frac{1}{p}\left(2^{(p-9) / 4}\left(C_{N-1}-3 C_{n}\right) \pm 2 U h\right) \\
& \quad \equiv 2^{(p-1) / 4} B_{(p-1) / 2}\left(-U^{2} h+\frac{2}{3} U^{2}-\frac{Q_{2}}{2}\right) \pm h(h-1) U^{3}(\bmod p),
\end{aligned}
$$

where the sign \pm is chosen in such a way that the left-hand side is a $p$ integer, and $N=3(p-1) / 2, n=(p-1) / 2-1$.

Proof. We get this congruence using Theorem 2, by substitution into the congruence for a quadratic field from [2] and by rearranging modulo $p^{2}$. Note that the sums $\sum_{i=0}^{n-1}\left(\begin{array}{c}n \\ i\end{array}\right) \frac{C_{i}}{n-i}$ and the numbers $E_{n+1} /(n+1), W_{p}$ cancel each other by these rearrangements.

REMARK. The congruence in Corollary 1 can be rewritten in the form

$$
\begin{aligned}
& \frac{1}{p}\left(2^{(p-1) / 4}\left(\frac{1}{3} B_{3(p-1) / 2}-3 B_{(p-1) / 2}\right) \pm 2 U h\right) \\
& \equiv 2^{(p-1) / 4} B_{(p-1) / 2}\left(-U^{2} h+\frac{2}{3} U^{2}+2-\frac{Q_{2}}{2}\right) \pm h(h-1) U^{3}(\bmod p) .
\end{aligned}
$$

ExAmple. (i) If $p=29$ then $h=1, U=1 / 2, C_{41} \equiv 82(\bmod 841)$, $C_{13} \equiv 662(\bmod 841), Q_{2} \equiv 2(\bmod 29)$.

(ii) If $p=229$ then $h=3, U=1 / 2, C_{341} \equiv 32702(\bmod 52441), C_{113} \equiv$ $27206(\bmod 52441), Q_{2} \equiv 68(\bmod 229)$.

\section{References}

[1] E. R. Hansen, A Table of Series and Products, Prentice-Hall, 1973.

[2] S. Jakubec, Congruence of Ankeny-Artin-Chowla type modulo $p^{2}$ for cyclic fields of prime degree l, Acta Arith. 74 (1996), 293-310.

[3] —, The congruence for Gauss's period, J. Number Theory 48 (1994), 36-45.

[4] —, On Vandiver's conjecture, Abh. Math. Sem. Univ. Hamburg 64 (1994), 105-124.

Matematický ústav SAV

Štefánikova 49

81473 Bratislava, Slovakia

E-mail: jakubec@mau.savba.sk 